\title{
TRATAMIENTO ENDOSCÓPICO DEL REFLUJO VESICOURETERAL EN PEDIATRÍA: EXPERIENCIA PRELIMINAR CON LA INYECCIÓN SUBURETERAL DE COAPTITE@.
}

\author{
Miguel Julián Mora Durbán*2, Francisco Javier Navarro Sebastián', María Belén Muñoz \\ Delgado*3, Jesús Isidoro García González y Pedro Dionisio Paniagua Andrés?.
}

Servicio de Urología' del Hospital General de Móstoles. Ex-miembro* del Servicio de Urología del Hospital General de Móstoles. Servicio de Urologíaª del Hospital Germans Trias i Pujol. Badalona. Barcelona. Servicio de Urología ${ }^{3}$ del Hospital General La Mancha Centro. Alcázar de San Juan. Ciudad Real. España.

\begin{abstract}
Resumen.- OBJETIVO: Analizar nuestra experiencia en el tratamiento endoscópico del reflujo vesicoureteral (RVU) en pediatría, mediante la inyección subureteral de hidroxiapatita cálcica (Coaptite®).

MÉTODOS: La serie incluye trece niños de edades entre los 6 y 13 años. La inyección endoscópica subureteral de Coaptite $\AA$ se aplicó en 20 unidades ureterales (UU) que presentaban RVU grados I-IV. EI RVU fue unilateral en 6 pacientes (46\%) y bilateral en 7 (54\%). Hubo 7 casos primarios y 6 secundarios a disfunción vesical y duplicidad ureteral.
\end{abstract}

RESULTADOS: El tiempo medio de seguimiento fue de 19 meses (6-28 meses). Se logró la corrección del RVU tras una inyección endoscópica en 15 casos (75\%). Una segunda inyección corrigió el RVU en 2 de otras

Miguel Julián Mora Durbán Rambla de Cataluña, 1 08007 Barcelona. (España) miguelimora@terra.es

Trabajo recibido: 19 de diciembre 2005
4 UU (50\%) tratadas, con lo que la cifra global de curaciones ascendió al 85\%. Esta tasa de curación se obtuvo tanto en los procedimientos que trataron casos primarios (13), como en las intervenciones por RVU secundario (7). Un $77 \%$ de pacientes no presentaron complicaciones, si bien el 23\% tuvieron molestias lumbares e hipogástricas autolimitadas al primer día tras el procedimiento. En 2 niños se detectó infección urinaria en el postoperatorio.

CONCLUSIONES: El tratamiento endoscópico del RVU con Coaptite ${ }^{\circledR}$ es una técnica sencilla y bien tolerada en niños. El $85 \%$ de los casos, tanto primarios como secundarios, curaron con mínima morbilidad.

Palabras clave: Reflujo vesicoureteral. Inyección endoscópica subureteral. Hidroxiapatita cálcica.

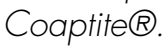

Summary.- OBJECTIVE: To review our experience in the endoscopic treatment of vesicoureteral reflux (VUR) in children with the subureteral injection of calcium hydroxy-

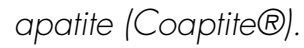

METHODS: The serie includes thirteen children whose age ranged between 6 and 13 years. The Coaptite $\AA$ implant was injected subureterally to 20 ureteral units (UU) affected by VUR grades I-IV. VUR was unilateral in six patients (46\%), bilateral in seven (54\%). There were 7 primary cases and 6 secondary to bladder dysfunction and duplex systems.

RESULTS: Mean follow-up was 19 months (6-28 months). VUR was cured in $75 \%$ of cases after a single injection. A second injection resolved 2 out of 4 treated UU (50\%) rendering a global success rate of $85 \%$. This cure rate was achieved for 13 and 7 procedures treating primary and secondary VUR, respectively. $77 \%$ of patients reported 
no adverse events with only $23 \%$ of them complaining of transient mild lumbar and hypogastric discomfort limited to the first postoperative day. In 2 children postoperative urinary infection was detected.

CONCLUSIONS: Endoscopic subureteral injection with Coaptite $\AA$ is a simple technique well tolerated in children. In $85 \%$ of primary and secondary cases cure was achieved with minimal morbidity.

Keywords: Vesicoureteral reflux. Endoscopic subureteral injection. Calcium hydroxyapatite.

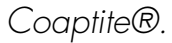

\section{INTRODUCCIÓN}

La asociación entre reflujo vesicoureteral (RVU) e infección del tracto urinario (ITU) ha sido claramente establecida (1). Como consecuencia de la combinación de hiperpresión sobre el riñón e ITU, se producen cambios pielonefríticos, capaces de causar insuficiencia renal e hipertensión en un número importante de pacientes $(2,3)$.

EI RVU se trata habitualmente de 2 formas: Conservadora, controlando la ITU con antibióticos, hasta la resolución del reflujo; y quirúrgicamente, cuando se trata de grados altos donde la tasa de curación espontánea es baja $(4,5)$.

Desde que en 1981, la inyección submucosa de pasta de politetrafluoruro de etileno (Teflon®) por debajo del orificio ureteral afecto, se demostró útil en la eliminación del RVU (6), este procedimiento ha ganado popularidad, utilizándose cada vez con más frecuencia $(7,8)$. Sin embargo, algunas complicaciones del teflón, como la formación de granulomas y la emigración a otros órganos, han propiciado la introducción de nuevos productos para el implante endoscópico $(9,10)$. Polidimetilsiloxano, un elastómero sólido derivado de la silicona (Macroplastique $($ ), colágeno bovino purificado (Zyplast $($ )), y dextranomero-copolímero del ácido hialurónico (Deflux®), aparecen como las 3 substancias no autólogas más utilizadas, sobre las que hidroxiapatita cálcica (Coaptite $\left.{ }^{\circledR}\right)$, ofrece ventajas de biocompatibilidad y ausencia de mutagenicidad (11-14). Con cifras globales de éxito alrededor del $80 \%$, la polémica sobre que tipo de sustancia es más idónea y cuál es la durabilidad de estos tratamientos aún continúa en la actualidad $(15,16)$.

Coaptite $\AA$, es un implante biocompatible compuesto de microesferas sintéticas de hidroxiapati- ta cálcica suspendidas en un gel acuoso de glicerina, desarrollado para el tratamiento endoscópico permanente de la incontinencia urinaria y el $\operatorname{RVU}(17,18)$. Describimos nuestra experiencia con la inyección endoscópica submeática (IESM) de Coaptite $₫$ sobre 20 unidades ureterales (UU) en un grupo de 13 pacientes pediátricos con RVU grado I-IV y un seguimiento medio post-cirugía de 19 meses.

\section{PACIENTES Y MÉTODOS}

Desde junio de 2003 hasta abril de 2005, fueron intervenidos mediante esta técnica 13 pacientes pediátricos (9 niñas y 4 niños), con edades entre 6 y 13 años, diagnosticados de RVU unilateral 16 casos) o bilateral ( 7 casos), tanto primario como secundario, totalizando $20 \mathrm{UU}$ tratadas.

El estudio diagnóstico incluyó siempre urocultivos periódicos, ecografía, cistouretrografía miccional (CUMS) y gammagrafía renal (DMSA). En algunos pacientes, se realizó también urografía cuando existió indicación clínica. Sólo en 4 casos se realizo estudio urodinámico pre-cirugía ante una alta sospecha de inestabilidad del detrusor o disinergia (incoordinación). En tres de ellos se confirmó la inestabilidad y se administró tratamiento anticolinérgico durante 12 meses, sin que mejorase el reflujo. El grado del RVU se estableció de acuerdo a la clasificación del Comité Internacional para el estudio del RVU (19). La indicación de cirugía endoscópica tuvo como criterio clave el fracaso de tratamiento conservador: ITU recurrente bajo profilaxis antibiótica, incremento de la severidad, persistencia del RVU, o empeoramiento morfo-funcional renal. Consideramos contraindicación para la técnica el RVU grado V, la presencia de ITU en el momento de la cirugía y la patología vesical o ureteral concomitante (divertículo paraureteral) con indicación de cirugía abierta.

Después de la evaluación preoperatoria y obtener consentimiento informado escrito de los padres/tutores en todos los casos, los pacientes fueron hospitalizados y tratados mediante IESM bajo anestesia general. Con un uretrocistoscopio pediátrico Storz ${ }^{\circledR} 9.5,11$ y $14 \mathrm{Fr}$ y óptica de $30^{\circ}$, se exploró la localización, número, y aspecto de los orificios ureterales, así como cualquier otra alteración endoscópica asociada. La inyección se realizó mediante jeringa pre-cargada con $1 \mathrm{cc}$. de Coaptite $\AA$ (BioForm Medical, Franksville, Wisconsin, USA) conectada a un cateter 3.7 FR insertado a través del canal de trabajo del cistoscopio y provisto de una aguja 21.5 $G$ (Figura 1). Una cantidad variable pero no inferior a 0.25 cc de Coaptite ${ }^{\circledR}$ fue inyectada a unos 3-4 $\mathrm{mm}$ por debajo del borde inferior del orificio ureteral 
refluyente, puncionando la mucosa y dirigiendo la aguja hacia el tunel submucoso de la unión uretero-vesical, hasta sobreelevar y obliterar parcialmente el meato (Figura 2). A criterio del endoscopista, se pudo repetir la punción para lograr la localización y cantidad deseada del implante, confirmándose la ausencia de extravasación y permeabilidad del orificio antes de finalizar el procedimiento. En los casos de bilateralidad, ambos meatos fueron tratados en la misma sesión. La vejiga no se dejó cateterizada y el alta hospitalaria fue habitualmente al día siguiente de la intervención.

Los criterios de valoración del resultado postoperatorio han sido: Resolución del RVU (curación, mejoría o fracaso), tolerabilidad y complicaciones. Los controles postcirugía en los primeros 12

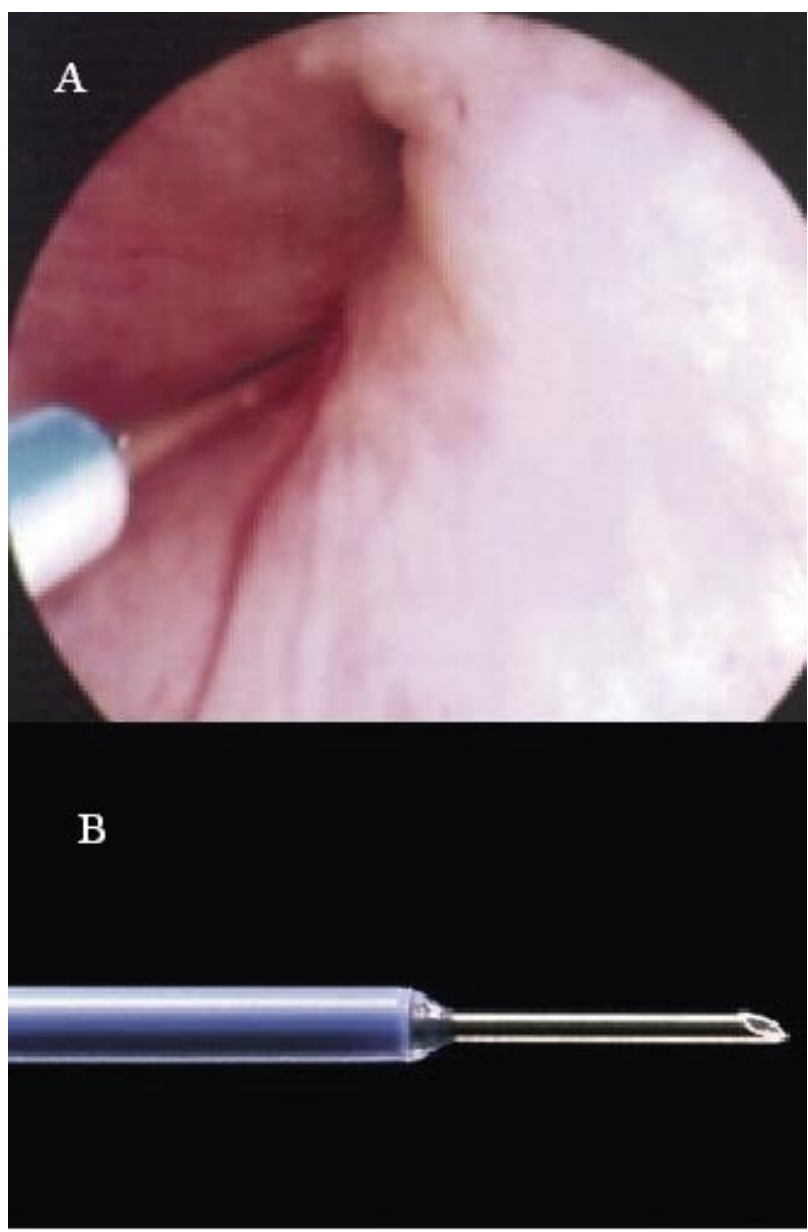

FIGURA 1. Al Cistoscopia. La aguja penetra hasta la submucosa vesical introducida a las 6 del horario cistoscópico a unos 4 milímetros distal al meato ureteral refluyente.

B) Punta de la aguja 21.5G para la inyección endoscópica. meses de seguimiento incluyeron anamnesis dirigida sobre síntomas miccionales, episodios de ITU, dolor lumbar, fiebre, etc., así como analítica de sangre y orina, pruebas de función renal, ecografía abdominal y CUMS en los primeros 3-6 meses, que se repitió al año en los casos de curación. La profilaxis antibiótica se mantuvo hasta constatar la eliminación del reflujo. En los pacientes que persistió el RVU tras el primer control, se ofertó realizar re-tratamiento pasados al menos 3 meses del implante. El resultado definitivo se evidenció con una $2^{a}$ CUMS/Gammagrafía renal pasados al menos 6 meses de la primera. La curación clínica se definió como la desaparición completa de los síntomas y ausencia de RVU tras el primer tratamiento constatada mediante CUMS. Se consideró mejoría, la atenuación del RVU preexistente a un grado I, activo y asintomático. El fracaso se definió como

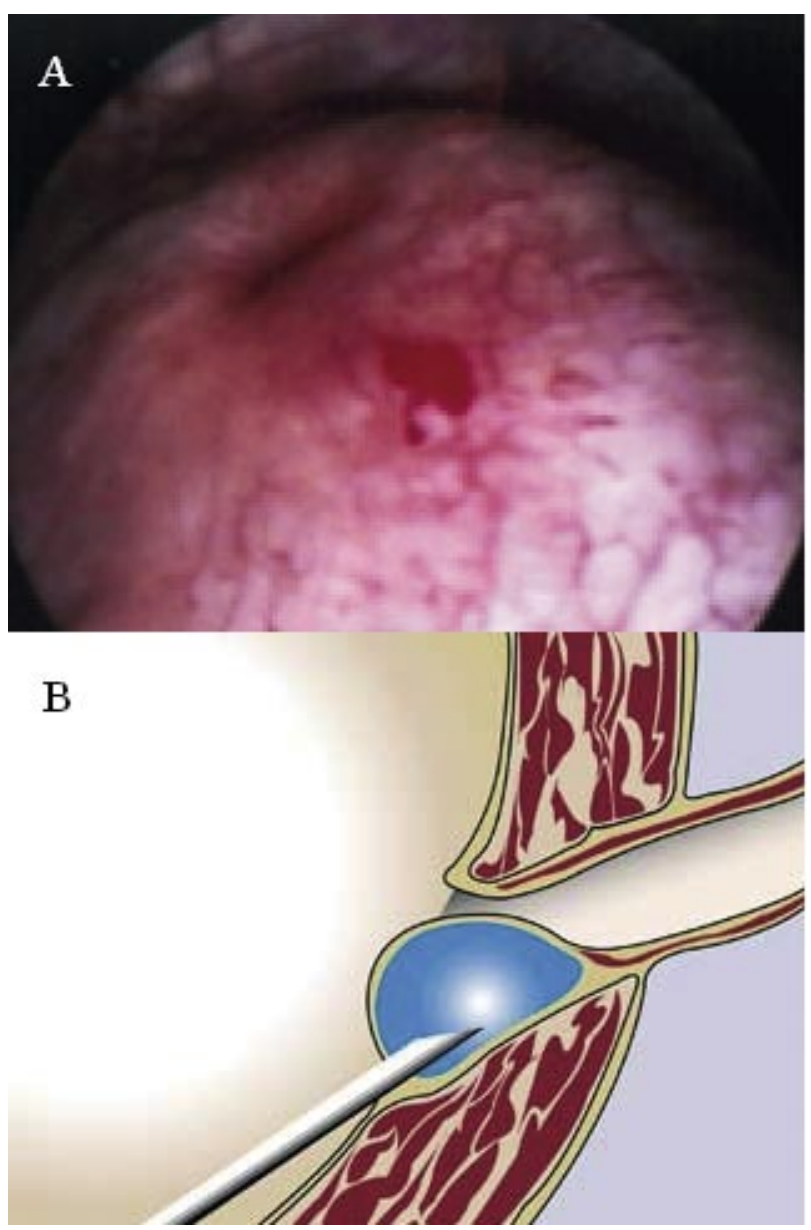

FIGURA 2. Al Cistoscopia. Aspecto del orificio ureteral una vez inyectado el material protésico. Tras la inyección el meato queda sobreelevado en forma de "cráter de volcán" por el efecto compresivo del implante.

B) Esquema gráfico de la localización subureteral del implante inyectable. 
la persistencia del RVU tras el tratamiento documentada por CUMS. Los pacientes que tras 24 meses de seguimiento permanecieron asintomáticos y se consideraron curados, sobre la base de los resultados de urocultivos, CUMS y pruebas de morfo-función renal, fueron dados de alta para una última revisión a los 48 meses.

\section{RESULTADOS}

Nuestro análisis incluyó 13 pacientes pediátricos (20 UU), de los que 9 eran niñas $(69 \%)$, todos con un tiempo mínimo de evaluación y tratamiento conservador precirugía de al menos 18 meses. La mediana de edad fue 10.5 años (rango 6-13 años). Siete pacientes presentaban afectación bilateral $(54 \%)$, con una discreta mayor proporción del lado derecho (55\%, 11 UU). Hubo 6 niños (46\%) con RVU secundario: 3 por disfunción funcional vesical ( 4 UU afectas), y 3 por duplicidad ureteral (3 UU afectas).

El habón submeático se obtuvo mediante la inyección de un volumen medio $0.60 \mathrm{cc}$ (rango: 0.25-1.50 ml) de Coaptite $®$, considerándose que el implante fue adecuado y el resultado endoscópico idóneo, en el $100 \%$ de las UU. Todos los pacientes pudieron ser dados de alta a las 24 horas de la intervención. El tiempo medio de seguimiento postimplante ha sido de 19 meses (rango: 6-28 meses). La CUMS postoperatoria mostró resolución completa del RVU en 15 UU (75\%) tras la $1^{a}$ inyección. Las respuestas estratificadas según el grado de reflujo se presentan en la Tabla I.

El porcentaje de curaciones según el grado de reflujo después de la primera inyección fue del $100 \%$ para los grado I (4 UU), $77 \%$ en grado II $(9$
UU), y $67 \%$ en III (6 UU). Tratamos un único caso grado IV, que obtuvo mejoría. En 4 UU $(20 \%)$, se realizó una $2^{a}$ inyección, con mejoría del RVU en 2 , mientras que otras $2 \mathrm{UU}$ han quedado en observación, sin profilaxis, con un reflujo grado I activo y asintomático. Ninguno de estos 2 últimos casos ha presentado complicaciones ni nuevas infecciones tras un seguimiento de 12 y 14 meses respectivamente. La cifra total de curaciones para el global de la serie obtenida tras la repetición de la inyección en estos 4 casos fue del $85 \%$. La tasa de curación final del reflujo grado II fue del $88 \%$ y del $83 \%$ en el grado III. El único caso grado IV, tras 2 inyecciones, obtuvo una mejoría significativa con reducción a grado I activo, asintomático, que no requirió tratamiento antibiótico profiláctico ulterior. En ningún caso se precisó una $3^{\underline{a}}$ inyección, aunque hay dos pacientes que pudieran necesitarlo en un futuro.

De los 13 procedimientos que trataron RVU primario, se obtuvo curación en 11 (85\%), con mejoría en $2(15 \%)$. Hubo 7 intervenciones en las que se trató un RVU secundario, consiguiéndose la curación en $6(86 \%)$ y mejoría en 1 (14\%), siendo la patología subyacente disfunción funcional vesical en 4 procedimientos ( 3 curaciones y 1 mejoría) así como duplicidad pieloureteral en 3 ( 3 curaciones).

Globalmente, la IESM no obtuvo curación en 3 casos (15\%), donde persistió un RVU grado I tras el procedimiento, si bien, estos pacientes permanecieron asintomáticos sin profilaxis antibiótica, con seguimientos de 6, 12 y 14 meses.

Ninguno de los pacientes presentó complicaciones importantes tras el procedimiento y cuando aparecieron, fueron de escasa significación clínica, como molestias lumbares $\mathrm{y} / \mathrm{o}$ hipogástricas autoli-

TABLA I. RESPUESTA AL TRATAMIENTO SEGÚN EL GRADO DE REFLUJO.

\begin{tabular}{|c|c|c|c|c|}
\hline \multirow{2}{*}{$\begin{array}{l}\text { RVU } \\
\text { Grado }\end{array}$} & \multirow{2}{*}{$\begin{array}{l}\text { Unidades } \\
\text { Ureterales }\end{array}$} & \multicolumn{2}{|c|}{ Curación (\% acumulado) } & \multirow[t]{2}{*}{ Mejoría (\%) } \\
\hline & & Tras $1^{\mathrm{a}}$ inyección & Tras $2^{\mathrm{a}}$ inyección & \\
\hline $\mathrm{I}$ & 4 & $4(100)$ & - & - \\
\hline II & 9 & $7(77)$ & $8(88)$ & $1(12)^{*}$ \\
\hline III & 6 & $4(67)$ & $5(83)$ & $1(17)^{\star *}$ \\
\hline IV & 1 & - & - & $1(100)^{* *}$ \\
\hline TOTAL & 20 & $15(75)$ & $17(85)$ & $3(15)$ \\
\hline
\end{tabular}

*Tras una inyección.

** Tras dos inyecciones. 
mitadas a los primeros días de postoperatorio en 3 niños $(23 \%)$. Se detectó ITU en el seguimiento de 2 pacientes (15\%), ambos con buena respuesta al tratamiento ambulatorio prescrito. En ningún caso hubo aparición de ureteropielectasia, ni otros síntomas que pudiesen indicar alergia, intolerancia local o migración del implante. En 1 caso se objetivó RVU contralateral de novo. En los casos con re-tratamiento, la cistoscopia en el $2^{\circ}$ procedimiento, mostró déficit de sustancia de abultamiento en la parte externa del meato. Ningún paciente requirió cirugía abierta tras el implante por fracaso y/o complicaciones secundarias al tratamiento endoscópico.

En los controles radiológicos diferidos, debemos señalar que dada la naturaleza mineral del implante, este se detecta como una imagen de densidad ósea sobre el área pélvica. Esta imagen, al ser sugestiva de litiasis, puede confundir, siendo clave en su diagnóstico diferencial, el antecedente de la inyección endoscópica de Coaptite ${ }^{\circledR}$.

\section{DISCUSIÓN}

Cualquier sustancia que utilicemos como implante inyectable, debe tener un buen perfil de seguridad, ser estable e inerte localmente, permaneciendo en el lugar de inyección sin degradación ni migración a otros órganos, capaz de mantener el volumen inyectado durante largo tiempo, así como fácil de utilizar con los catéteres y agujas endoscópicas habituales, a la vez que lo suficientemente viscosa para evitar su extravasado desde el lugar de aplicación $(14,20)$. En nuestra experiencia, los aspectos mas ventajosos de Coaptite $\AA$ para el tratamiento mediante IESM fueron su facilidad de aplicación, eficacia en la corrección del RVU a bajo volumen de inyección, a la vez que una excelente biocompatibilidad y ausencia de complicaciones.

El procedimiento endoscópico resultó técnicamente sencillo. Coaptite $\AA$ tiene una escasa viscosidad obviando la necesidad de un inyector de alta presión y permitió utilizar agujas mínimamente traumáticas facilitando puntos clave del procedimiento. Para Herz (21) existe un claro efecto de curva de aprendizaje $y$, en un grupo sin experiencia previa con este tratamiento, comunica un $46 \%$ de éxitos al inicio del estudio mientras que posteriomente, duplica esta cifra con sólo una inyección. Sin embargo, no sucedió así en nuestra serie y la ausencia de diferencias significativas en los resultados durante las fases inicial y final del estudio, estuvo probablemente relacionada con la selección de pacientes, excluyendo niños con reflujo grado $V$ y previamente intervenidos, más que por una experiencia previa con agentes in- yectables (22). Así, nuestros resultados se alinean con los de Dodat (23) que comunicó un éxito uniforme independiente del grado de reflujo.

La cifra global de curación tras la primera inyección fue del $75 \%$ y con la repetición de una segunda IESM, ascendió al $85 \%$. También en pacientes pediátricos, Frey y Michael, reportan resultados ligeramente mejores con pasta de teflón y polidimetilxilosano, mientras que los resultados con colágeno-glutaraldehido son similares a los nuestros $(12,24)$. Por el contrario, en una reciente revisión sistemática de la literatura, de la Peña y colaboradores (25), no encuentran diferencias ente las distintas substancias analizadas para la inyección (politetrafluoruro de etileno, pilimetilxilosano y dextranómero del copolímero de ácido hialurónico) en RVU grados I-III. Puri (7), utilizando teflón como material de inyección, comunicó cifras muy superponibles a las nuestras, con $76 \%$ de éxitos tras una sola inyección y del $85 \%$ con múltiples inyecciones, si bien, con un seguimiento a 10 años.

Comparados a las tasas de curación tras cirugía abierta, nuestros resultados se muestran claramente inferiores. Así, la Asociación Americana de Urología en su Panel de Directrices para el tratamiento del RVU primario, señala cifras de éxito del $98 \%$ para grados IV o menores (4). Aún con recientes innovaciones técnicas, los mejores resultados utilizando este procedimiento comunican cifras de curación rara vez superiores al $90 \%$ para grados IIIV (26). En cambio, en la revisión de de la Peña (25), el manejo endoscópico fue igual de efectivo que la cirugía abierta y con menores efectos secundarios. Por tanto, debemos considerar las ventajas en términos de invasividad operatoria, menor hospitalización y posibilidad de reintervención ( 1 de cada 5 procedimientos en nuestra serie), al sopesar ambas formas de tratamiento. Además, ya que el RVU puede desaparecer espontáneamente en pacientes con bajo grado, el tratamiento quirúrgico resulta aquí controvertido (27). Sin embargo, hasta la desaparición del RVU pueden pasar años con tratamiento antibiótico continuado que, en si mismo, es motivo de posibles complicaciones, sin olvidar las visitas repetidas al hospital y molestias para pacientes y familia. Por lo que, sabiendo que los grados bajos de RVU muestran tasas de corrección altas con la IESM, su atractivo es indudable. Así, en un estudio de las preferencias de los padres de niños con RVU, la profilaxis fue la opción elegida como tratamiento inicial, pero si este se prolongaba durante 3 ó 4 años, la mayoría prefirió una corrección definitiva. Aunque el tratamiento endoscópico era menos efectivo, fue preferido frente a la cirugía abierta, presumiblemente, por que la corrección mediante IESM resultaba menos invasiva (28). 
Aunque es conocido el fracaso de esta forma de tratamiento asociado a la existencia de alteraciones funcionales de la micción $(13,21)$, nosotros obtuvimos una respuesta favorable en la mayoría de intervenciones en las que se trató RVU secundario. En una reciente comunicación utilizando Deflux ${ }^{\circledR}$, Pérez-Brayfield (29), describe un $68 \%$ de éxito en pacientes complejos, entre los que incluye pacientes con duplicidad ureteral, reflujo persistente después de cirugía abierta, vejiga neurógena, ureter ectópico y divertículo periureteral, si bien, el seguimiento estuvo limitado a tres meses. En una serie analizando el tratamiento endoscópico de RVU complejo, Argüelles (30), obtiene éxitos superiores al $80 \%$ en 74 pacientes con diversas patologías asociadas y propone esta técnica como el tratamiento de elección inicial.

En los 4 casos que falló el tratamiento endoscópico, se hizo cistoscopia y se comprobó que el habón creado con él implante era insuficiente para producir una coaptación adecuada del meato, siempre por déficit en la porción externa del mismo, mientras que la porción interna siempre había quedado correctamente abultada. Desafortunadamente, la cistoscopia repetida tras cirugía no se realizó de rutina para determinar la posición del implante en todos los niños. Por tanto, la certeza de estos hallazgos clínicos depende de nuestra experiencia anecdótica con sólo aquellos pacientes en los que se repitió la cistoscopia e inyección por fallo de la técnica. En el estudio de Diamond, sobre 57 pacientes en los que el reflujo no fue corregido endoscópicamente, el desplazamiento del habón se plantea como la causa más frecuente del fracaso en el $35 \%$ de casos, aunque la anatomía de la vejiga indudablemente también jugaría un papel determinante (31). La apariencia y localización del habón del implante inmediatamente después de su inyección, adoptando una forma de volcán, es para algunos clínicos, el mejor pronóstico de éxito (32).

\section{RESULTADOS}

El porcentaje de curaciones según el grado de reflujo después de la primera inyección fue del $100 \%$ para los grado I (4 UU), $77 \%$ en grado II (9 UU), y $67 \%$ en III (6 UU). Tratamos un único caso grado IV, que obtuvo mejoría. En 4 UU $(20 \%)$, se realizó una $2^{\underline{a}}$ inyección, con curación del RVU en 2 , mientras que otras 2 UU obtuvieron mejoría, quedando un reflujo grado I activo y asintomático sin profilaxis. Ninguno de estos 2 últimos casos ha presentado complicaciones ni nuevas infecciones tras un seguimiento de 12 y 14 meses respectivamente. La cifra total de curaciones para el global de la serie obtenida tras la repetición de la inyección en estos 4 casos fue del $85 \%$. La tasa de curación final del reflujo grado II fue del $88 \%$ y del $83 \%$ en el grado III. El único caso grado IV, tras 2 inyecciones, obtuvo una mejoría significativa con reducción a grado I activo, asintomático, que no requirió tratamiento antibiótico profiláctico ulterior. En ningún caso se precisó una $3^{\underline{a}}$ inyección, aunque hay dos pacientes que pudieran necesitarlo en un futuro.

\section{CONCLUSIONES}

Nuestros resultados confirman que la IESM de Coaptite ${ }^{\circledR}$ es una alternativa viable frente a la cirugía abierta para el tratamiento del reflujo en niños, alineándose con los obtenidos por otras sustancias de abultamiento de contrastada eficacia. La mayoría de los casos (85\%), tanto primarios como secundarios, curaron con mínima morbilidad y corta estancia hospitalaria. Nuestro seguimiento actual, deja aún pendiente de confirmar su eficacia a largo plazo.

\section{BIBLIOGRAFÍA y LECTURAS RECOMENDADAS (*lectura de interés $y^{* *}$ lectura fundamental)}

1. SARGENT, M.A.: "Opinion. What is the normal prevalence of vesicouretral reflux?". Pediatr. Radiol., 30: 587, 2000.

2. OLBING, H.: CLAISSON, I.; ABEL, K.D. y cols.: "Renal scars and parenchymal thinning in children with vesicoureteral reflux: a 5-year report of the international reflux study in children (European branch)". J. Urol., 148: 1653, 1992.

3. BAILEY, R.R.; MAILING, T.M.J.; SWAINSON, C.P.: "Vesicoureteral reflux and reflux nephropaty". Schrier R.W., Gottschilk C.W., editors. Disease of the kidney. 5th ed. p. 689-727. Little-Brown; Boston, 1993.

*4. ELDER, J.S.: "Guidelines for consideration for surgical repair of vesicoureteral reflux". Curr. Opin. Urol., 10: 579, 2000.

5. MOURIQUAND, P.D.E.: "Surgical treatment of vesicoureteral reflux". Spitz L., Coran A.G., editors. Rob \& Smith Operative Surgery: Pediatric Surgery. p. 638-42. Butterworth; London, 1995.

6. MATOUSCHEK, E.: "Die behandlung des vesikorenalen Refluxes durch transureterale Einspritzung von polytetrafluoroethylenepaste". Urologe, 20: 263, 1981.

7. PURI, P.: "Ten-year experience with subureteric Teflon (polytyetrafluoroethylene) injection (STING) in the treatment of vesicoureteral reflux”. Br. J. Urol., 75: 126, 1995. 
*8. MORAN PENCO, J.M.; GOMEZ FRAILE, A.; RODRIGUEZ ALARCON, J. y cols.: "Evolution of the treatment of vesicoureteral reflux in Spain". J Urol., 171: 834, 2004.

9. ARAGONA, F.; D'URSO, L.; SCREMIN, E. y cols.: "Polytetrafluoroethylene giant granuloma and adenopathy: long-term complications following subureteral polytetrafluoroethylene injection for the treatment of vesicoureteral reflux in children". J. Urol., 158: 1539, 1997.

10. AARONSON, I.A.; RAMES, R.A.; GREENE, W.B. y cols.: "Endoscopic treatment of reflux: migration of Teflon to the lungs and brain". Eur. Urol., 23: 394, 1993.

11. PURI, P.; CHERTIN, B.; MURUGESH, V.; DASS, L.: "Treatment of vesicoureteral reflux by endoscopic injection of dextranomer/hyaluronic acid copolymer: Preliminary results". J. Urol., 170: 1541, 2003.

12. FREY, P.; BERGER, D.; JENNY, P. y cols.: "Subureteral collagen injection for the endoscopic treatment of vesicoureteral reflux in children. Follow-up study of 97 treated ureters and histological analysis of collagen implants". J. Urol., 148: $718,1992$.

13. ABOUTALEB, H.; BOLDUC, S.; UPADHYAY, J. y cols.: "Subureteral polydimethylsiloxane injection versus extravesical reimplantation for primary low grade vesicoureteral reflux in children: A comparative study". J. Urol., 169: 313, 2003.

14. SOLOMON, L.Z.; BIRCH, B.R.; COOPER, A.J. y cols.: "Nonhomologous bioinjectable materials in urology: 'size matters'?’. BJU Int., 85: 641, 2000.

15. CHERTIN, B.; COLHOUN, E.; MURUGESH, V. y cols.: "Endoscopic treatment of vesicoureteral reflux. An eleven to seventeen years follow up". J Urol., 167: 1443, 2002.

16. KEATING, M.: "Role of periureteral injections in children with vesicoureteral reflux". Current Opinion in Urology, 15: 369, 2005.

17. MEVORACH, R.; RABINOWITZ, R.; BECK, C. y cols.: "Endoscopic treatment of vesicoureteral reflux with Coaptite: the first 50 patients". American Urologic Association Meeting, [podium \#429]. Orlando, FL, 2002.

18. MAYER, R.; LIGHTFOOT, M.; JUNG, I.: "Preliminary evaluation of calcium hydroxylapatite as a transurethral bulking agent for stress urinary incontinence". Urology, 57: 434, 2001.

19. LEBOWITZ, R.L.; OLBING, H.; PARKKULAINEN, K.V. y cols.: "International system of radiographic grading of vesicoureteric reflux". Pediatr. Radiol., 15: 105, 1985.

20. SUGIYAMA, T.; HANAI, T.; HASHIMOTO, K. y cols.: "Long-term outcome of the endoscopic correction of vesico-ureteric reflux: a comparison of injected substances”. BJU Int., 94: 381, 2004.
21. HERZ, D.; HAFEZ, A.; BÄGLI, D. y cols.: "Efficacy of endoscopic subureteral macroplastique injection for the treatment of vesicoureteral reflux in children: a North American clinical report". J. Urol., 166: 1880, 2001.

22. MARTÍNEZ-PIÑEIRO, L.; HERNANDEZ, A.; MORA, M.J. y cols.: "Tratamiento del reflujo vesicoureteral en adultos mediante inyección endoscópica de pasta de teflón”. Arch. Esp. Urol., 41: 53, 1988.

23. DODAT, H.; VALMALLE, A.F.; WEIDMANN, J.D. y cols.: "Endoscopic treatment of vesicoureteral reflux in children. Five-year assessment of the use of Macroplastique ${ }^{\circledR}$ ”. Prog. Urol., 8: $1001,1998$.

24. MICHAEL, V.; DAVARIS, P.; ARHONTAKIS, A. y cols.: "Effects of submucosal Teflon paste injection in vesicoureteric reflux: results with 1- and 2-year follow-up data". Eur. Urol., 23: 379, 1993.

*25. DE LA PEÑA ZARZUELO, E.: "Tratamiento del reflujo vésico-ureteral primario en la infancia: Comparación de dos revisiones sitemáticas". Actas Urol. Esp., 29: 138, 2005.

**26. KIRSCH, A.J.; PEREZ-BRAYFIELD, M.; SMITH, E.A. y cols.: "The modified sting procedure to correct vesicoureteral reflux: improved results with submucosal implantation within the intramuralureter". J Urol., 171: 2413, 2004.

27. WHEELER, D.; VIMALACHANDRA, D.; HODSON, E.M. y cols.: "Antibiotics and surgery for vesicoureteric reflux: a meta-analysis of randomised controlled trials". Arch. Dis. Child., 88: 688, 2003.

28. OGAN, K.; POHL, H.G.; CARLSON, D. y cols.: "Parental preferences in the management of vesicoureteral reflux". J. Urol., 166: 244, 2001.

**29. PEREZ-BRAYFIELD, M.; KIRSCH, A.J.; HENSLE, T.W. y cols.: "Endoscopic treatment with dextranomer/hyaluronic acid for complex cases of vesi-coureteral reflux". J. Urol., 172: 1614, 2004.

*30. ARGÜELLES SALIDO, E.; GARCÍA MERINO, F.; MILLAN LOPEZ, A. y cols.: "Reflujo vesicoureteral complejo. Revisión de nuestra serie". Actas Urol. Esp., 29: 685, 2005.

31. DIAMOND, D.A.; CALDAMONE, A.A.; BAUER, S.B. y cols.: "Mechanisms of failure of endoscopic treatment of vesicoureteral reflux based on endoscopic anatomy". J. Urol., 170: 1556, 2003.

*32. LAVELLE, M.T.; CONLIN, M.J.; SKOOG, S.J.: "Subureteral injection of Deflux for correction of reflux: analysis of factors predicting success". Urology, 65: 564, 2005.

33. VEREECKEN, R.L.; PROESMANS, W.: "Results of endoscopic treatment for vesico-ureteric reflux". Eur. Urol., 27: 76, 1995. 